\title{
Can We Cluster ICU Treatment Strategies for Traumatic Brain Injury by Hospital Treatment Preferences?
}

Iris E. Ceyisakar ${ }^{1,2^{*}} \mathbb{D}$, Jilske A. Huijben ${ }^{1}$, Andrew I. R. Maas², Hester F. Lingsma and Nikki van Leeuwen ${ }^{1}$ CENTER-

TBI participants and investigators

(c) 2021 The Author(s)

\begin{abstract}
Background: In traumatic brain injury (TBI), large between-center differences in treatment and outcome for patients managed in the intensive care unit (ICU) have been shown. The aim of this study is to explore if European neurotrauma centers can be clustered, based on their treatment preference in different domains of TBI care in the ICU.

Methods: Provider profiles of centers participating in the Collaborative European Neurotrauma Effectiveness Research in TBI study were used to assess correlations within and between the predefined domains: intracranial pressure monitoring, coagulation and transfusion, surgery, prophylactic antibiotics, and more general ICU treatment policies. Hierarchical clustering using Ward's minimum variance method was applied to group data with the highest similarity. Heat maps were used to visualize whether hospitals could be grouped to uncover types of hospitals adhering to certain treatment strategies.
\end{abstract}

Results: Provider profiles were available from 66 centers in 20 different countries in Europe and Israel. Correlations within most of the predefined domains varied from low to high correlations (mean correlation coefficients 0.2-0.7). Correlations between domains were lower, with mean correlation coefficients of 0.2. Cluster analysis showed that policies could be grouped, but hospitals could not be grouped based on their preference.

Conclusions: Although correlations between treatment policies within domains were found, the failure to cluster hospitals indicates that a specific treatment choice within a domain is not a proxy for other treatment choices within or outside the domain. These results imply that studying the effects of specific TBI interventions on outcome can be based on between-center variation without being substantially confounded by other treatments.

Trial registration: We do not report the results of a health care intervention.

Keywords: Provider profiling, Between-hospital variation, Comparative effectiveness research, Traumatic brain injury

\section{Introduction}

Traumatic brain injury (TBI) remains a major global health issue, being one of the leading causes of mortality

*Correspondence: iris.kohen@gmail.com

${ }^{1}$ Center for Medical Decision Making, Department of Public Health, Erasmus Medical Center, PO Box 2040, 3000, CA, Rotterdam, The Netherlands

Full list of author information is available at the end of the article and disability with 2.5 million reported cases each year within the European Union and United Kingdom [1-3].

The primary injury is irreversible, and the main focus of treatment is on avoiding and limiting secondary brain damage. In patients with severe TBI, this is often informed by intracranial pressure (ICP) or brain-metabolic monitoring. Previous studies have debated monitoring and treatment choices in TBI [4], and evidence 
underpinning monitoring and treatment recommendations is relatively weak.

This uncertainty is reflected in large between-center differences in processes and outcomes for patients treated in the intensive care unit (ICU) after TBI $[5,6]$. The differences in treatment policy can be exploited to study treatment effectiveness in comparative effectiveness research (CER). One approach to CER is to identify the most effective treatment, by comparing hospitals' treatment choices and relating these to their outcomes. In recent years, this approach has gained popularity in TBI as a complementary approach to the evidence base provided by randomized controlled trials [7]. CER can be used to identify a causal relationship between a treatment and outcome if known and unknown confounders can be adequately adjusted for and if the treatment under investigation is not correlated with other treatment policies. To date, it is unknown whether certain treatment strategies in patients with TBI are related. Such knowledge would be essential when comparing outcomes on a hospital level within the framework of CER to study whether differences in outcomes can be attributed to the separate interventions.

If, on the other hand, multiple treatment choices are correlated, it gives the possibility to group these together and identify hospitals with, for example, a more aggressive treatment strategy. Conclusions could then only be drawn on a very general level: whether a more aggressive or a more passive treatment strategy is more effective. Within the framework of CER, however, this would make it impossible to study specific treatments and their effect on outcome because some specific treatment aspects within the strategy may be beneficial and others even harmful.

Focusing on the domains of ICP monitoring, prophylactic antibiotics, transfusion targets, and general ICU management, our aim was to investigate correlations between treatment policies and to explore if European neurotrauma centers can be clustered based on their treatment strategy in patients with TBI.

\section{Methods \\ Collaborative European Neurotrauma Effectiveness Research in TBI Study}

The Collaborative European NeuroTrauma Effectiveness Research in TBI (CENTER-TBI) study is a prospective longitudinal multicenter observational study conducted across Europe and Israel (ClinicalTrials.gov ref. NCT02210221) [8]. CENTER-TBI aims to better characterize and describe TBI in a European context and to further advance the care of patients with TBI within the broader international framework of the International Initiative for TBI Research (https://intbir.nih.gov/).
Principal investigators of each participating center in this study received questionnaires about the structures (type of facilities and equipment, the qualifications of medical staff and their organizations) and processes (treatment policies in different phases of TBI care) of their center: the provider profiling questionnaires [9]. Participants were explicitly asked for their general policy rather than for individual treatment preferences. General policy was defined as "the way the large majority of patients $(>75 \%)$ with a certain indication would be treated." Detailed information about the content, development, and validation of the original 321 questions can be found in an earlier publication [9]. Baseline characteristics for centers were described using frequencies and percentages.

\section{Predefined Treatment Domains}

We selected 58 questions on the basis of expert consensus concerning care in the ICU setting, covering the domains of coagulation and transfusion, neurosurgery, ICP monitoring, prophylactic antibiotics, and general management. The selected questions were chosen before the analysis on the basis of clinical relevance. Follow-up, conditional questions ("if you answered A, then specify...") and all open questions were excluded, aiming to obtain a standardized overview of treatment approaches.

Questions from predefined domains were chosen to enable stratification of hospitals over multiple domains. To determine possible underlying treatment strategies, polychoric correlation coefficients between questions were calculated [10]. Correlations were visualized with correlation plots, using only absolute values (between 0 and 1), as any negative correlations were as relevant as positive correlations in determining treatment strategies. Missing answers were disregarded for calculation of the correlation. Of the 58 questions, 44 questions were complete, and the other 12 had up to 8 out of 66 answers missing. We looked at correlations of questions within the predefined domains (exploring consistency in treatment policies within a specific domain) as well as correlations between the domains (exploring interdependencies of treatments between domains).

\section{Regrouping of Questions}

After correlations were determined, the questions were grouped based on the data, ignoring the previously defined domains, with an hierarchical cluster analysis using Ward's minimum variance method, to group together the questions with the highest similarity [11]. This is an agglomerative clustering method in which the 
data points (questions) are clustered (across all domains) in different steps until only questions with the greatest similarity form a cluster. We used the Bayesian inference criterion for $k$-means to determine the number of clusters that were to be formed $[12,13]$.

\section{Heat Maps}

By using the same clustering algorithm, heat maps were made for each newly formed group of questions. Heat maps allow for the recognition of patterns in the preference of hospitals and made it possible to determine whether we could discern certain types of hospitals. This was performed on all complete cases: centers with missing answers within the cluster of questions were disregarded. For cluster two, 53 of the 66 participating hospitals were included in the heat map; for cluster five, 47 of the hospitals were included, and all other clusters included all participating hospitals. All analysis were performed in $\mathrm{R}$ version 3.3.0 using the following packages: pheatmap, RColorBrewer, foreign, cluster, corrplot, dplyr, and fmsb [14-21].

\section{Results CENTER-TBI Study}

Provider profiling questionnaires were completed in 66 centers (97\% response rate), mainly by intensivists $(n=33,50 \%)$ and neurosurgeons $(n=23,35 \%)$, but otherwise by administrative staff $(n=11,17 \%)$, neurologists $(n=5,8 \%)$, anesthesiologists $(n=5,8 \%)$, and a trauma surgeon $(n=1,2 \%)$. The majority of these centers had an academic affiliation $(n=60,91 \%)$. The center characteristics are described in Supplementary Table 1 and in more detail in a previous publication [9].

\section{Correlation within and Between Domains}

Correlation between treatment policies within the predefined domains was variable (Fig. 1a-e). Correlations within the domain of prophylactic antibiotics (mean correlation coefficient $=0.6$, range $0.4-0.8$ ) ranged from moderate to strong, but questions were based on only one very specific topic. The correlation within the other domains was shown to be much lower (Table 1). Correlations between domains were lower, with mean correlation coefficients of 0.2 for each domain correlated with all other domains (Table 1 and Fig. 1f).

\section{Data-Driven Cluster Analysis}

The cluster analysis revealed four clusters, one fewer compared with the clinically determined domains (Fig. 2). The grouping remained very similar to the predefined domains, especially for the original domain of neurosurgery. A few questions did correlate with other subdomains, mainly due to overlap in topic of the questions. For example, the question, "Is a coagulation panel assessed prior to insertion of an ICP monitoring device?" could span the domains of coagulation and transfusion as well as ICP monitoring (Supplementary Table 2).

\section{Grouping of Hospitals}

No hospital types were discernable in the heat maps made for each cluster of questions (Fig. 3, for the heat map of all the questions see Suppl. Figure 2). The heat maps served as a visual indication of the possibility to cluster the hospitals. Based on the visualization of these data, we have decided that further clustering should not be done. Although most similar questions had been grouped together, heat maps showed no clear pattern in the preferences of the hospitals, indicating that they could not be grouped based on their treatment tendencies.

\section{Discussion}

This study aimed to group European neurotrauma centers into clusters based on their treatment preference in patients with TBI in a variety of domains. Hospitals could not be clustered based on their reported choices of treatment within the five domains of the provider profiling questionnaires. These results imply that it is unlikely that hospitals can be categorized as a certain type of hospital based on the treatment strategies they follow across multiple domains of monitoring and treatment in patients with TBI.

The lack of evident treatment policies across multiple domains might be explained by a lack of strong evidence of the effectiveness of certain treatments, leading to weak guideline recommendations, which may cause heterogeneity in treatment strategies across Europe [22]. However, it could also be the result of more individualized medicine, in which case treatment strategies are based on the individual patient and monitoring characteristics [1]. TBI is a complex heterogeneous syndrome that might not be captured with a single treatment strategy. With the advanced monitoring devices and the range of brain and system targeted therapies available, variation between centers in treatment strategies is likely.

For future statistical analyses, our finding that the questions correlated mainly within their previously defined subdomain implies that all elements of TBI treatment can, and have to, be analyzed separately rather than combining different domains when relations between treatment and outcome are explored. The correlation of some questions with questions from a different domain could be attributed to overlap in the subject of the questions. 


\section{Correlations between questions of the 5 predefined domains}
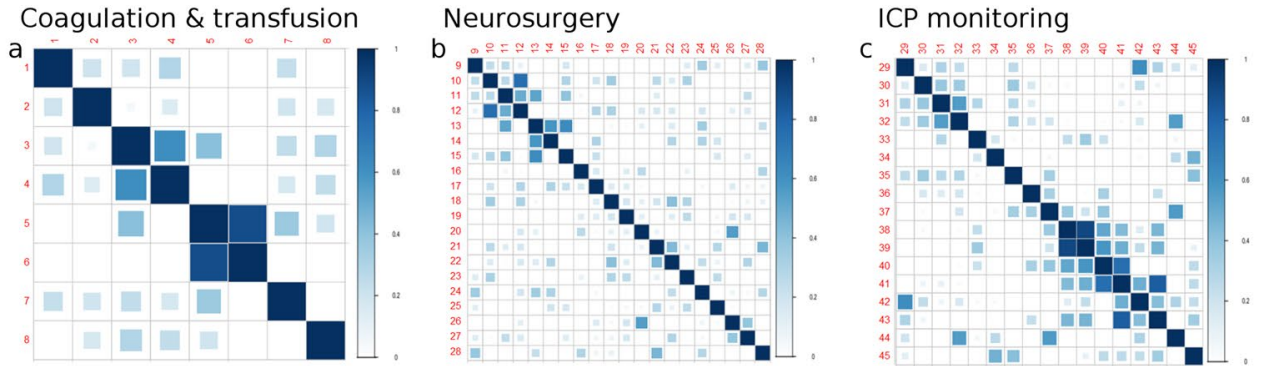

Prophylactic antibiotics

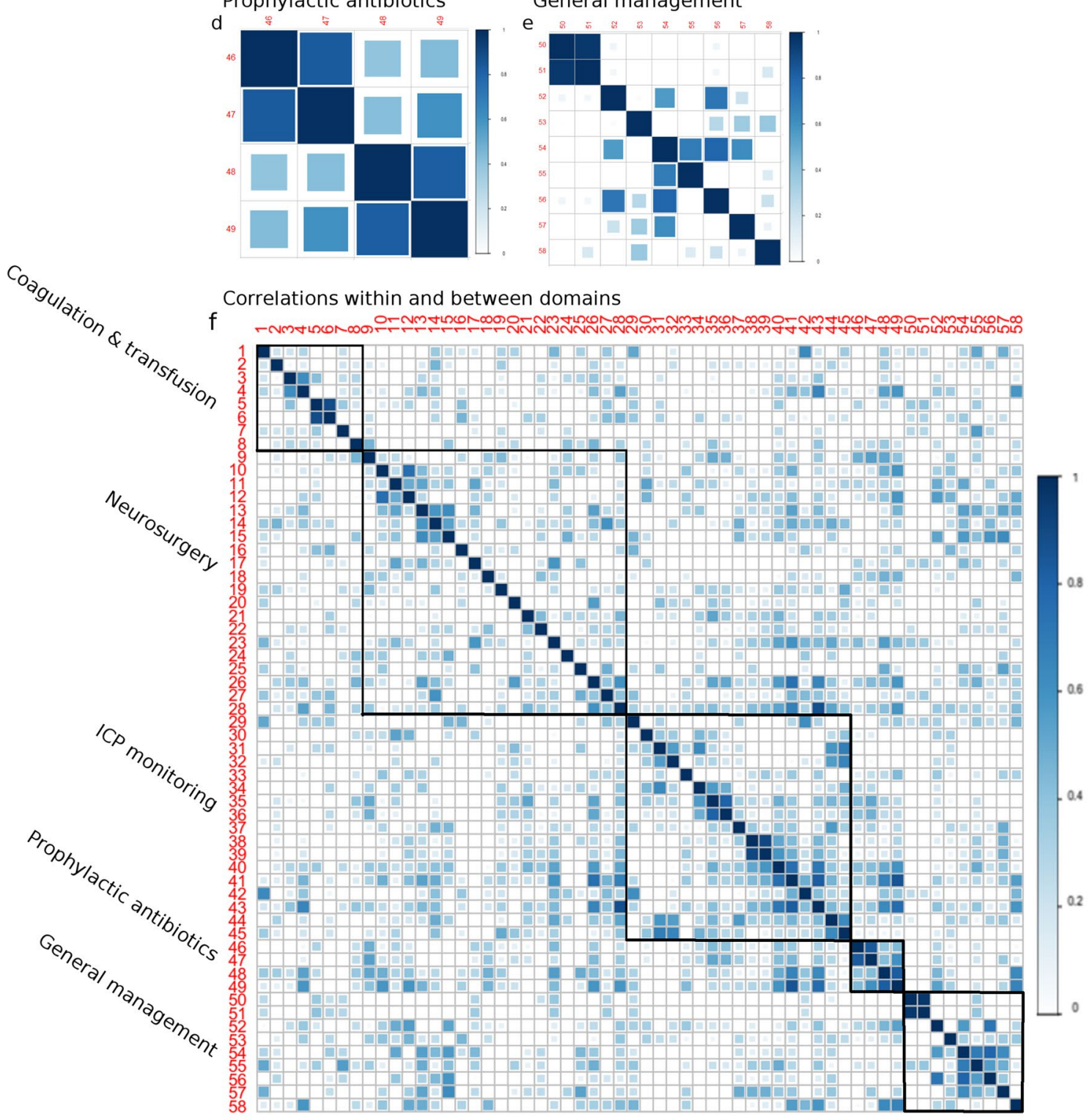

General management

Fig. 1 Correlation plot showing correlations between questions, grouped (squares) to show the five predefined domains: intracranial pressure (ICP) monitoring (a), coagulation and transfusion $(\mathbf{b})$, surgery $(\mathbf{c})$, prophylactic antibiotics $(\mathbf{d})$, and more general ICU treatment policies (e), and correlations within and between the predefined domains (f). The correlations were calculated with Pearson correlations, and a higher correlation is visualized as a darker blue. ICU intensive care unit 
Table 1 Overview of the correlation coefficient calculated for questions within and between predefined domains

\begin{tabular}{|c|c|c|c|c|}
\hline Domain & Mean (SD) & Min & Max & $\begin{array}{l}\text { Number } \\
\text { of ques- } \\
\text { tions }\end{array}$ \\
\hline \multicolumn{5}{|c|}{ Correlation coefficients for correlation between questions within the predefined domains } \\
\hline Coagulation \& transfusion targets & $0.2(0.2)$ & 0 & 0.9 & 8 \\
\hline Neurosurgery & $0.2(0.2)$ & 0 & 0.8 & 19 \\
\hline ICP monitoring & $0.3(0.2)$ & 0 & 0.9 & 17 \\
\hline Prophylactic antibiotics & $0.6(0.2)$ & 0.4 & 0.8 & 4 \\
\hline General management & $0.3(0.2)$ & 0 & 1 & 9 \\
\hline \multicolumn{5}{|c|}{ Correlation coefficients for questions between the predefined domains } \\
\hline Coagulation \& transfusion targets versus rest & $0.2(0.1)$ & 0 & 0.7 & \\
\hline Neurosurgery versus rest & $0.2(0.2)$ & 0 & 0.9 & \\
\hline ICP monitoring versus rest & $0.2(0.2)$ & 0 & 0.9 & \\
\hline Prophylactic antibiotics versus rest & $0.2(0.2)$ & 0 & 0.9 & \\
\hline General management versus rest & $0.2(0.1)$ & 0 & 0.7 & \\
\hline
\end{tabular}

We used the Pearson correlation coefficient to determine correlations within domains (upper panel) and between domains (lower panel)

ICP intracranial pressure, Max maximum, Min minimum, SD standard deviation

Other reasons for correlations with other domains could be dependent on who is responsible for the decisions being made: for example, decisions for treatment of the patient are made by the neurosurgeon would have a higher chance of correlating with other decisions made by that neurosurgeon.

Based on our study, we may conclude that future CER analyses will be likely to measure a direct effect of one intervention on outcome instead of a general effect of multiple treatment effects. This is important knowledge to continue CER research within TBI, in which outcomes between centers are compared to find underlying differences in treatment. Although unmeasured confounders will always have to be considered, knowing that multiple treatments are not interdependent is a first step in further elucidating the effects of treatment choices. This study has its strengths and limitations. This study was conducted in multiple neurotrauma centers across Europe. The development and dissemination of the questionnaires was done in different phases. Two methods were used to determine relations between and within certain treatment strategy domains. With hierarchical cluster analyses, we confirmed the results of correlation analyses. However, our study also has its limitations; in a survey study using provider profiling questionnaires, centers only indicate their treatment strategy and do not provide an objective measure of real-time practice. This could overestimate or underestimate the use of general policies. The centers included in this study are mostly academic medical centers, and a more heterogeneous group of care providers could have potentially shown a clearer division in hospital types. Previous studies from CENTER-TBI show that, even within the sample of mostly academic centers, substantial practice variation exists [23-25].

Further, the study is focused on hospitals in Europe, and it is possible that these findings cannot be extrapolated to other large regions, such as the United States.

Possibly better suited for the purpose of grouping hospitals would be a questionnaire that is more specific. Future research using a more detailed questionnaire might be a solution to increase reliability of indicated treatment preferences. More targeted questions could allow for a better and more thorough understanding. This would give insight into why decisions are made and by whom. However, the better we understand and the more specific the information is, the harder it will be to visualize, generalize, and simplify enough to be able to present it graphically.

This is the first study that studied underlying relations in treatment strategies, and these results need to be confirmed in other studies.

\section{Conclusions}

We found correlations in treatment policies within domains, especially for neurosurgical interventions, but no evidence that hospitals could be clustered, indicating that a specific treatment choice within a domain is not a proxy for other treatment choices within or outside the domain. Because we did not find an indication that some centers, in general, were more eager to treat or reach higher treatment intensity levels overall, future TBI analyses should be conducted per specific treatment item 


\section{questions grouped based on hierarchical cluster analysis}

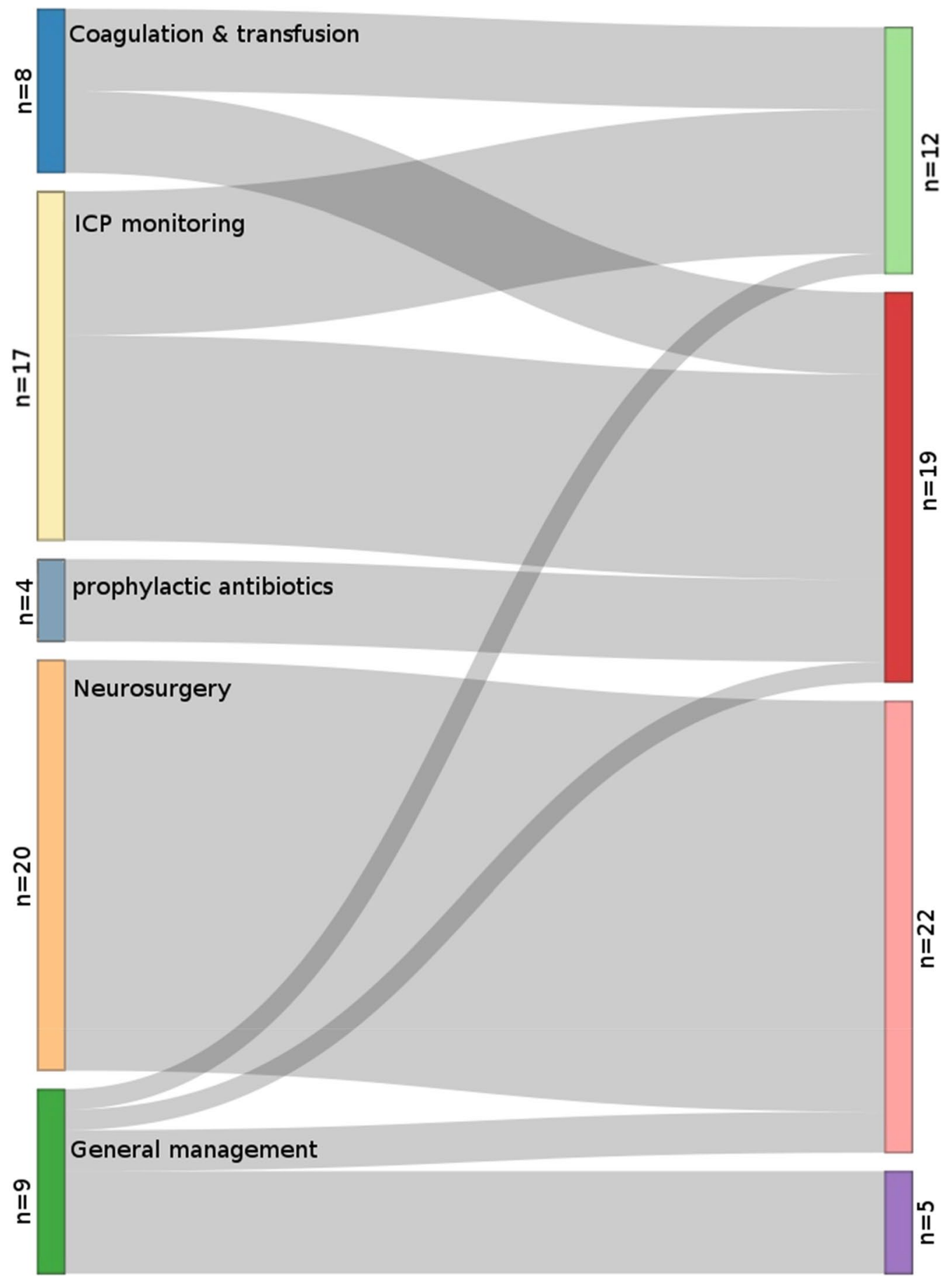

Fig. 2 Sankey diagram showing regrouping of questions according to hierarchical clustering. On the left, questions are grouped according to what was decided to be clinically relevant. On the right, the questions are regrouped, and the shifting of questions is visualized, with a thicker gray line indicating a larger number of questions. ICP, intracranial pressure 


\section{Heatmaps for identification of hospital types}
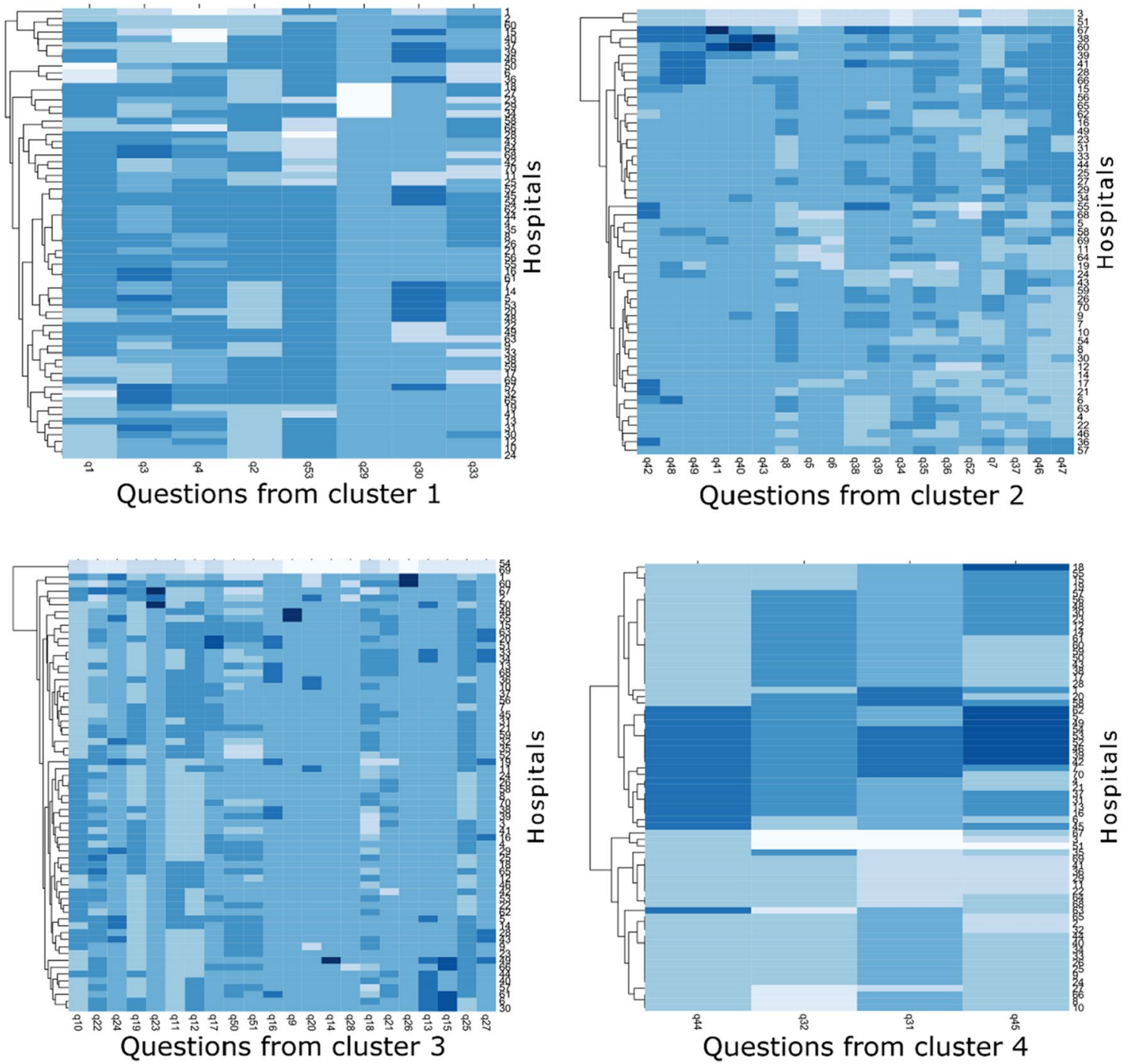

Fig. 3 Heat maps showing similarities between hospitals in answers given to questions within the previously determined clusters of questions. This was based on the hierarchical cluster analyses. The colors in the heat map relate to the specific answers: within a column, the same color indicates that two hospitals have given the same answer to the corresponding question. ICP intracranial pressure

instead of per treatment domain. Furthermore, within the CER paradigm, this implies that analyzing effects of an intervention on outcome is likely to measure a direct effect of that intervention without being substantially confounded by a general effect of multiple treatments.

\section{Supplementary Information}

The online version contains supplementary material available at https://doi. org/10.1007/s12028-021-01386-y.

\section{Author details}

${ }^{1}$ Center for Medical Decision Making, Department of Public Health, Erasmus Medical Center, PO Box 2040, 3000, CA, Rotterdam, The Netherlands. ${ }^{2}$ Department of Neurosurgery, Antwerp University Hospital, University of Antwerp, Drie Eikenstraat 655, 2650 Antwerp, Belgium.

\section{Acknowledgments}

The authors thank all clinical and research staff at the CENTER-TBI sites for completing the provider profiling questionnaires. Data used in preparation of this manuscript were obtained in the context of CENTER-TBI, a large collaborative project with the support of the European Commission 7th Framework 
program (602150). The funder had no role in the design of the study and collection, analysis, and interpretation of data and in writing the manuscript.

The CENTER-TBI participants and investigators: Cecilia Åkerlund ${ }^{1}$, Krisztina Amrein ${ }^{2}$, Nada Andelic ${ }^{3}$, Lasse Andreassen ${ }^{4}$, Audny Anke ${ }^{5}$, Anna Antoni ${ }^{6}$, Gérard Audibert ${ }^{7}$, Philippe Azouvi ${ }^{8}$, Maria Luisa Azzolini ${ }^{9}$, Ronald Bartels ${ }^{10}$, Pál Barzó $^{11}$, Romuald Beauvais ${ }^{12}$, Ronny Beer ${ }^{13}$, Bo-Michael Bellander ${ }^{14}$, Antonio Belli ${ }^{15}$, Habib Benali ${ }^{16}$, Maurizio Berardino ${ }^{17}$, Luigi Beretta ${ }^{9}$, Morten Blaabjerg ${ }^{18}$, Peter Bragge ${ }^{19}$, Alexandra Brazinova ${ }^{20}$, Vibeke Brinck ${ }^{21}$, Joanne Brooker ${ }^{22}$, Camilla Brorsson ${ }^{23}$, Andras Buki ${ }^{24}$, Monika Bullinger ${ }^{25}$, Manuel Cabeleira ${ }^{26}$, Alessio Caccioppola ${ }^{27}$, Emiliana Calappi ${ }^{27}$, Maria Rosa Calvi ${ }^{9}$, Peter Cameron ${ }^{28}$, Guillermo Carbayo Lozano ${ }^{29}$, Marco Carbonara ${ }^{27}$, Simona Cavallo ${ }^{17}$, Giorgio Chevallard $^{30}$, Arturo Chieregato ${ }^{30}$, Giuseppe Citerio ${ }^{31}, 32$, Hans Clusmann ${ }^{33}$, Mark Coburn $^{34}$, Jonathan Coles ${ }^{35}$, Jamie D. Cooper ${ }^{36}$, Marta Correia ${ }^{37}$, Amra Čović ${ }^{38}$, Nicola Curry ${ }^{39}$, Endre Czeiter ${ }^{24}$, Marek Czosnyka ${ }^{26}$, Claire Dahyot-Fizelier ${ }^{40}$, Paul Dark ${ }^{41}$, Helen Dawes ${ }^{42}$, Véronique De Keyser ${ }^{43}$, Vincent Degos ${ }^{16}$, Francesco Della Corte ${ }^{44}$, Hugo den Boogert ${ }^{10}$, Bart Depreitere ${ }^{45}$, Đula Đilves ${ }^{46}$, Abhishek Dixit $^{47}$, Emma Donoghue ${ }^{22}$, Jens Dreier ${ }^{48}$, Guy-Loup Dulière ${ }^{49}$, Ari Ercole ${ }^{47}$, Patrick Esser ${ }^{42}$, Erzsébet Ezer ${ }^{50}$, Martin Fabricius ${ }^{51}$, Valery L. Feigin ${ }^{52}$, Kelly Foks ${ }^{53}$, Shirin Frisvold ${ }^{54}$, Alex Furmanov ${ }^{55}$, Pablo Gagliardo ${ }^{56}$, Damien Galanaud ${ }^{16}$, Dashiell Gantner ${ }^{28}$, Guoyi Ga ${ }^{57}$, Pradeep George ${ }^{58}$, Alexandre Ghuysen ${ }^{59}$, Lelde Giga $^{60}$, Ben Glocker ${ }^{61}$, Jagoš Golubovic ${ }^{46}$, Pedro A. Gomez ${ }^{62}$, Johannes Gratz ${ }^{63}$, Benjamin Gravesteijj ${ }^{64}$, Francesca Grossi ${ }^{44}$, Russell L. Gruen ${ }^{65}$, Deepak Gupta ${ }^{66}$, Juanita A. Haagsma ${ }^{64}$, lain Haitsma ${ }^{67}$, Raimund Helbok ${ }^{13}$, Eirik Helseth ${ }^{68}$, Lindsay Horton ${ }^{69}$, Jilske Huijben ${ }^{64}$, Peter J. Hutchinson ${ }^{70}$, Bram Jacobs $^{71}$, Stefan Jankowski ${ }^{72}$, Mike Jarrett ${ }^{21}$, Ji-yao Jiang ${ }^{58}$, Faye Johnson ${ }^{73}$, Kelly Jones ${ }^{52}$, Mladen Karan $^{46}$, Angelos G. Kolias ${ }^{70}$, Erwin Kompanje ${ }^{74}$, Daniel Kondziella ${ }^{51}$, Evgenios Kornaropoulos ${ }^{47}$, Lars-Owe Koskinen ${ }^{75}$, Noémi Kovács ${ }^{76}$, Ana Kowark ${ }^{77}$, Alfonso Lagares $^{62}$, Linda Lanyon ${ }^{58}$, Steven Laureys ${ }^{78}$, Fiona Lecky 79,80 , Didier Ledoux ${ }^{78}$, Rolf Lefering ${ }^{81}$, Valerie Legrand ${ }^{82}$, Aurelie Lejeune ${ }^{83}$, Leon Levi ${ }^{84}$, Roger Lightfoot $^{85}$, Hester Lingsma ${ }^{64}$, Andrew I.R. Maass ${ }^{43}$, Ana M. Castaño-León ${ }^{62}$, Marc Maegele ${ }^{86}$, Marek Majdan ${ }^{20}$, Alex Manara ${ }^{87}$, Geoffrey Manley ${ }^{88}$, Costanza Martino ${ }^{89}$, Hugues Maréchal ${ }^{49}$, Julia Mattern ${ }^{90}$, Catherine McMahon ${ }^{91}$, Béla Melegh $^{92}$, David Menon ${ }^{47}$, Tomas Menovsky ${ }^{43}$, Ana Mikolic ${ }^{64}$, Benoit Misset ${ }^{78}$, Visakh Muraleedharan ${ }^{58}$, Lynnette Murray ${ }^{28}$, Ancuta Negru' ${ }^{93}$, David Nelson' ${ }^{1}$, Virginia Newcombe ${ }^{47}$, Daan Nieboer ${ }^{64}$, József Nyirádi ${ }^{2}$, Otesile Olubukola ${ }^{79}$, Matej Oresic ${ }^{94}$, Fabrizio Ortolano ${ }^{27}$, Aarno Palotie ${ }^{95,96,97}$, Paul M. Parizel ${ }^{98}$, Jean-François Payen ${ }^{99}$, Natascha Perera ${ }^{12}$, Vincent Perlbarg ${ }^{16}$, Paolo Persona ${ }^{100}$, Wilco Peul ${ }^{101}$, Anna Piippo-Karjalainen ${ }^{102}$, Matti Pirinen ${ }^{95}$, Dana Pisica ${ }^{64}$, Horia Ples ${ }^{93}$, Suzanne Polinder ${ }^{64}$, Inigo Pomposo ${ }^{29}$, Jussi P. Posti ${ }^{103}$, Louis Puybasset ${ }^{104}$, Andreea Radoi ${ }^{105}$, Arminas Ragauskas ${ }^{106}$, Rahul Raj ${ }^{102}$, Malinka Rambadagalla ${ }^{107}$, Isabel Retel Helmrich ${ }^{64}$, Jonathan Rhodes ${ }^{108}$, Sylvia Richardson ${ }^{109}$, Sophie Richter ${ }^{47}$, Samuli Ripatti $i^{95}$, Saulius Rocka ${ }^{106}$, Cecilie Roe ${ }^{110}$, Olav Roise ${ }^{111,112}$, Jonathan Rosand ${ }^{113}$, Jeffrey V. Rosenfeld ${ }^{114}$, Christina Rosenlund ${ }^{115}$, Guy Rosentha ${ }^{55}$, Rolf Rossaint ${ }^{77}$, Sandra Rossi ${ }^{100}$, Daniel Rueckert ${ }^{61}$ Martin Rusnák ${ }^{116}$, Juan Sahuquillo ${ }^{105}$, Oliver Sakowitz ${ }^{90,117}$, Renan Sanchez-Porras ${ }^{117}$, Janos Sandor ${ }^{118}$, Nadine Schäfer ${ }^{81}$, Silke Schmidt ${ }^{119}{ }^{\prime}$, Herbert Schoechl' ${ }^{120}$, Guus Schoonman ${ }^{121}{ }^{\prime}$ Rico Frederik Schou ${ }^{122}$, Elisabeth Schwendenwein ${ }^{6}$, Charlie Sewalt ${ }^{64}$, Ranjit D. Singh ${ }^{101}$, Toril Skandsen ${ }^{123,124}$, Peter Smielewski ${ }^{26}$, Abayomi Sorinola ${ }^{125}$, Emmanuel Stamatakis ${ }^{47}$, Simon Stanworth ${ }^{39}$, Robert Stevens ${ }^{126}$, William Stewart ${ }^{127}$, Ewout W. Steyerberg ${ }^{64,128}$, Nino Stocchetti ${ }^{129}$, Nina Sundström ${ }^{130}$, Riikka Takala ${ }^{131}$, Viktória Tamás ${ }^{125}$, Tomas Tamosuitis ${ }^{132}$, Mark Steven Taylor ${ }^{20}$, Braden $\mathrm{Te} \mathrm{Ao}^{52}$, Olli Tenovuo ${ }^{103}$, Alice Theadom ${ }^{52}$, Matt Thomas ${ }^{87}$, Dick Tibboel ${ }^{133}$, Marjolein Timmers ${ }^{74}$, Christos Tolias ${ }^{134}$, Tony Trapani ${ }^{28}$, Cristina Maria Tudora ${ }^{93}$, Andreas Unterberg ${ }^{90}$, Peter Vajkoczy ${ }^{135}$, ShirleyVallance ${ }^{28}$, Egils Valeinis ${ }^{60}$, Zoltán Vámos $^{50}$, Mathieu van der Jagt ${ }^{136}$, Gregory Van der Steen ${ }^{43}$, Joukje van der Naalt $^{71}$, Jeroen T.J.M. van Dijck ${ }^{101}$, Inge A. van Erp ${ }^{101}$, Thomas A. van Essen ${ }^{101}$, Wim Van Hecke ${ }^{137}$, Caroline van Heugten ${ }^{138}$, Dominique Van Praag ${ }^{139}$, Ernest van Veen ${ }^{64}$, Thijs Vande Vyvere ${ }^{137}$, Roel P. J. van Wijk ${ }^{101}$, Alessia Vargiolu ${ }^{32}$, Emmanuel Vega ${ }^{83}$, Kimberley Velt ${ }^{64}$, Jan Verheyden ${ }^{137}$, , Paul M. Vespa ${ }^{140}$, Anne Vik ${ }^{123,141}$, Rimantas Vilcinis ${ }^{132}$, Victor Volovicic7 ${ }^{67}$, Nicole von Steinbüche $\left.\right|^{38}$, Daphne Voormolen ${ }^{64}$, Petar Vulekovic ${ }^{46}$, Kevin K.W. Wang ${ }^{142}$, Daniel Whitehouse ${ }^{47}$, Eveline Wiegers ${ }^{64}$, Guy Williams ${ }^{47}$, Lindsay Wilson ${ }^{69}$, Stefan Winzeck ${ }^{47}$, Stefan Wolf ${ }^{143}$, Zhihui Yang ${ }^{113}$, Peter Ylén ${ }^{144}$, Alexander Younsi ${ }^{90}$, Frederick A. Zeiler $^{47,145}$, Veronika Zelinkova ${ }^{20}$, Agate Ziverte ${ }^{60}$, and Tommaso Zoerle ${ }^{27}$.

${ }^{1}$ Department of Physiology and Pharmacology, Section of Perioperative Medicine and Intensive Care, Karolinska Institutet, Stockholm, Sweden

2János Szentágothai Research Centre, University of Pécs, Pécs, Hungary

${ }^{3}$ Division of Surgery and Clinical Neuroscience, Department of Physical Medicine and Rehabilitation, Oslo University Hospital and University of Oslo, Oslo, Norway
${ }^{4}$ Department of Neurosurgery, University Hospital Northern Norway, Tromso, Norway

${ }^{5}$ Department of Physical Medicine and Rehabilitation, University Hospital Northern Norway, Tromso, Norway

${ }^{6}$ Trauma Surgery, Medical University Vienna, Vienna, Austria

${ }^{7}$ Department of Anesthesiology \& Intensive Care, University Hospital Nancy, Nancy, France

${ }^{8}$ Raymond Poincare hospital, Assistance Publique - Hopitaux de Paris, Paris, France

${ }^{9}$ Department of Anesthesiology \& Intensive Care, S Raffaele University Hospital, Milan, Italy

${ }^{10}$ Department of Neurosurgery, Radboud University Medical Center, Nijmegen, The Netherlands

${ }^{11}$ Department of Neurosurgery, University of Szeged, Szeged, Hungary

${ }^{12}$ International Projects Management, ARTTIC, Munchen, Germany

${ }^{13}$ Department of Neurology, Neurological Intensive Care Unit, Medical

University of Innsbruck, Innsbruck, Austria

${ }^{14}$ Department of Neurosurgery \& Anesthesia \& intensive care medicine,

Karolinska University Hospital, Stockholm, Sweden

${ }^{15} \mathrm{NIHR}$ Surgical Reconstruction and Microbiology Research Centre, Birmingham, UK

${ }^{16}$ Anesthesie-Réanimation, Assistance Publique - Hopitaux de Paris, Paris,

France

${ }^{17}$ Department of Anesthesia \& ICU, AOU Città della Salute e della Scienza di

Torino - Orthopedic and Trauma Center, Torino, Italy

${ }^{18}$ Department of Neurology, Odense University Hospital, Odense, Denmark

${ }^{19}$ BehaviourWorks Australia, Monash Sustainability Institute, Monash University, Victoria, Australia

${ }^{20}$ Department of Public Health, Faculty of Health Sciences and Social Work,

Trnava University, Trnava, Slovakia

${ }^{21}$ Quesgen Systems Inc., Burlingame, California, USA

${ }^{22}$ Australian \& New Zealand Intensive Care Research Centre, Department of Epidemiology and Preventive Medicine, School of Public Health and Preven-

tive Medicine, Monash University, Melbourne, Australia

${ }^{23}$ Department of Surgery and Perioperative Science, Umeå University,

Umeå, Sweden

${ }^{24}$ Department of Neurosurgery, Medical School, University of Pécs, Hungary and Neurotrauma Research Group, János Szentágothai Research Centre, University of Pécs, Hungary

${ }^{25}$ Department of Medical Psychology, Universitätsklinikum Hamburg-

Eppendorf, Hamburg, Germany

${ }^{26}$ Brain Physics Lab, Division of Neurosurgery, Dept of Clinical Neurosciences, University of Cambridge, Addenbrooke's Hospital, Cambridge, UK

${ }^{27}$ Neuro ICU, Fondazione IRCCS Cà Granda Ospedale Maggiore Policlinico, Milan, Italy

${ }^{28}$ ANZIC Research Centre, Monash University, Department of Epidemiology and Preventive Medicine, Melbourne, Victoria, Australia

${ }^{29}$ Department of Neurosurgery, Hospital of Cruces, Bilbao, Spain

${ }^{30}$ Neurolntensive Care, Niguarda Hospital, Milan, Italy

${ }^{31}$ School of Medicine and Surgery, Università Milano Bicocca, Milano, Italy

${ }^{32}$ Neurolntensive Care, ASST di Monza, Monza, Italy

${ }^{33}$ Department of Neurosurgery, Medical Faculty RWTH Aachen University, Aachen, Germany

${ }^{34}$ Department of Anesthesiology and Intensive Care Medicine, University Hospital Bonn, Bonn, Germany

${ }^{35}$ Department of Anesthesia \& Neurointensive Care, Cambridge University Hospital NHS Foundation Trust, Cambridge, UK

${ }^{36}$ School of Public Health \& PM, Monash University and The Alfred Hospital, Melbourne, Victoria, Australia

${ }^{37}$ Radiology/MRI department, MRC Cognition and Brain Sciences Unit, Cambridge, UK

${ }^{38}$ Institute of Medical Psychology and Medical Sociology, Universitätsmedizin Göttingen, Göttingen, Germany

${ }^{39}$ Oxford University Hospitals NHS Trust, Oxford, UK

${ }^{40}$ Intensive Care Unit, CHU Poitiers, Potiers, France

${ }^{41}$ University of Manchester NIHR Biomedical Research Centre, Critical Care Directorate, Salford Royal Hospital NHS Foundation Trust, Salford, UK

${ }^{42}$ Movement Science Group, Faculty of Health and Life Sciences, Oxford Brookes University, Oxford, UK

${ }^{43}$ Department of Neurosurgery, Antwerp University Hospital and University of Antwerp, Edegem, Belgium 
${ }^{44}$ Department of Anesthesia \& Intensive Care, Maggiore Della Carità Hospital, Novara, Italy

${ }^{45}$ Department of Neurosurgery, University Hospitals Leuven, Leuven, Belgium

${ }^{46}$ Department of Neurosurgery, Clinical centre of Vojvodina, Faculty of Medicine, University of Novi Sad, Novi Sad, Serbia

${ }^{47}$ Division of Anaesthesia, University of Cambridge, Addenbrooke's Hospital, Cambridge, UK

${ }^{48}$ Center for Stroke Research Berlin, Charité - Universitätsmedizin Berlin, corporate member of Freie Universität Berlin, Humboldt-Universität zu Berlin, and Berlin Institute of Health, Berlin, Germany

${ }^{49}$ Intensive Care Unit, CHR Citadelle, Liège, Belgium

${ }^{50}$ Department of Anaesthesiology and Intensive Therapy, University of Pécs, Pécs, Hungary

${ }^{51}$ Departments of Neurology, Clinical Neurophysiology and Neuroanesthesiology, Region Hovedstaden Rigshospitalet, Copenhagen, Denmark

${ }^{52}$ National Institute for Stroke and Applied Neurosciences, Faculty of Health and Environmental Studies, Auckland University of Technology, Auckland, New Zealand

${ }^{53}$ Department of Neurology, Erasmus MC, Rotterdam, the Netherlands

${ }^{54}$ Department of Anesthesiology and Intensive care, University Hospital Northern Norway, Tromso, Norway

${ }^{55}$ Department of Neurosurgery, Hadassah-hebrew University Medical center, Jerusalem, Israel

${ }^{56}$ Fundación Instituto Valenciano deNeurorrehabilitación (FIVAN), Valencia, Spain

${ }^{57}$ Department of Neurosurgery, Shanghai Renji hospital, Shanghai Jiaotong University/school of medicine, Shanghai, China

${ }^{58}$ Karolinska Institutet, INCF International Neuroinformatics Coordinating Facility, Stockholm, Sweden

${ }^{59}$ Emergency Department, $\mathrm{CHU}$, Liège, Belgium

${ }^{60}$ Neurosurgery clinic, Pauls Stradins Clinical University Hospital, Riga, Latvia

${ }^{61}$ Department of Computing, Imperial College London, London, UK

${ }^{62}$ Department of Neurosurgery, Hospital Universitario 12 de Octubre, Madrid, Spain

${ }^{63}$ Department of Anesthesia, Critical Care and Pain Medicine, Medical University of Vienna, Austria

${ }^{64}$ Department of Public Health, Erasmus Medical Center-University Medical Center, Rotterdam, The Netherlands

${ }^{65}$ College of Health and Medicine, Australian National University, Canberra, Australia

${ }^{66}$ Department of Neurosurgery, Neurosciences Centre \& JPN Apex trauma centre, All India Institute of Medical Sciences, New Delhi-1 10029, India

${ }^{67}$ Department of Neurosurgery, Erasmus MC, Rotterdam, the Netherlands

${ }^{68}$ Department of Neurosurgery, Oslo University Hospital, Oslo, Norway

${ }^{69}$ Division of Psychology, University of Stirling, Stirling, UK

${ }^{70}$ Division of Neurosurgery, Department of Clinical Neurosciences, Addenbrooke's Hospital \& University of Cambridge, Cambridge, UK

${ }^{71}$ Department of Neurology, University of Groningen, University Medical Center Groningen, Groningen, Netherlands

${ }^{72}$ Neurointensive Care, Sheffield Teaching Hospitals NHS Foundation Trust, Sheffield, UK

${ }^{73}$ Salford Royal Hospital NHS Foundation Trust Acute Research Delivery Team, Salford, UK

${ }^{74}$ Department of Intensive Care and Department of Ethics and Philosophy of Medicine, Erasmus Medical Center, Rotterdam, The Netherlands

${ }^{75}$ Department of Clinical Neuroscience, Neurosurgery, Umeå University, Umeå, Sweden

${ }^{76}$ Hungarian Brain Research Program - Grant No. KTIA_13_NAP-A-II/8, University of Pécs, Pécs, Hungary

${ }^{77}$ Department of Anaesthesiology, University Hospital of Aachen, Aachen, Germany

${ }^{78}$ Cyclotron Research Center, University of Liège, Liège, Belgium

${ }^{79} \mathrm{Centre}$ for Urgent and Emergency Care Research (CURE), Health Services Research Section, School of Health and Related Research (ScHARR), University of Sheffield, Sheffield, UK

${ }^{80}$ Emergency Department, Salford Royal Hospital, Salford UK

${ }^{81}$ Institute of Research in Operative Medicine (IFOM), Witten/Herdecke University, Cologne, Germany

${ }^{82} \mathrm{VP}$ Global Project Management CNS, ICON, Paris, France
${ }^{83}$ Department of Anesthesiology-Intensive Care, Lille University Hospital, Lille, France

${ }^{84}$ Department of Neurosurgery, Rambam Medical Center, Haifa, Israel

${ }^{85}$ Department of Anesthesiology \& Intensive Care, University Hospitals Southhampton NHS Trust, Southhampton, UK

${ }^{86}$ Cologne-Merheim Medical Center (CMMC), Department of Traumatology, Orthopedic Surgery and Sportmedicine, Witten/Herdecke University, Cologne, Germany

${ }^{87}$ Intensive Care Unit, Southmead Hospital, Bristol, Bristol, UK

${ }^{88}$ Department of Neurological Surgery, University of California, San Francisco, California, USA

${ }^{89}$ Department of Anesthesia \& Intensive Care,M. Bufalini Hospital, Cesena, Italy

${ }^{90}$ Department of Neurosurgery, University Hospital Heidelberg, Heidelberg, Germany

${ }^{91}$ Department of Neurosurgery, The Walton centre NHS Foundation Trust, Liverpool, UK

${ }^{92}$ Department of Medical Genetics, University of Pécs, Pécs, Hungary

${ }^{93}$ Department of Neurosurgery, Emergency County Hospital Timisoara, Timisoara, Romania

${ }^{94}$ School of Medical Sciences, Örebro University, Örebro, Sweden

${ }^{95}$ Institute for Molecular Medicine Finland, University of Helsinki, Helsinki, Finland

${ }^{96}$ Analytic and Translational Genetics Unit, Department of Medicine; Psychiatric \& Neurodevelopmental Genetics Unit, Department of Psychiatry; Department of Neurology, Massachusetts General Hospital, Boston, MA, USA

${ }^{97}$ Program in Medical and Population Genetics; The Stanley Center for Psy-

chiatric Research, The Broad Institute of MIT and Harvard, Cambridge, MA, USA

${ }^{98}$ Department of Radiology, University of Antwerp, Edegem, Belgium

${ }^{99}$ Department of Anesthesiology \& Intensive Care, University Hospital of Grenoble, Grenoble, France

${ }^{100}$ Department of Anesthesia \& Intensive Care, Azienda Ospedaliera Università di Padova, Padova, Italy

${ }^{101}$ Dept. of Neurosurgery, Leiden University Medical Center, Leiden, The Netherlands and Dept. of Neurosurgery, Medical Center Haaglanden, The Hague, The Netherlands

${ }^{102}$ Department of Neurosurgery, Helsinki University Central Hospital

${ }^{103}$ Division of Clinical Neurosciences, Department of Neurosurgery and

Turku Brain Injury Centre, Turku University Hospital and University of Turku, Turku, Finland

${ }^{104}$ Department of Anesthesiology and Critical Care, Pitié -Salpêtrière Teaching Hospital, Assistance Publique, Hôpitaux de Paris and University Pierre et Marie Curie, Paris, France

${ }^{105}$ Neurotraumatology and Neurosurgery Research Unit (UNINN), Vall

d'Hebron Research Institute, Barcelona, Spain

${ }^{106}$ Department of Neurosurgery, Kaunas University of technology and Vilnius University, Vilnius, Lithuania

${ }^{107}$ Department of Neurosurgery, Rezekne Hospital, Latvia

${ }^{108}$ Department of Anaesthesia, Critical Care \& Pain Medicine NHS Lothian \& University of Edinburg, Edinburgh, UK

${ }^{109}$ Director, MRC Biostatistics Unit, Cambridge Institute of Public Health, Cambridge, UK

${ }^{110}$ Department of Physical Medicine and Rehabilitation, Oslo University Hospital/University of Oslo, Oslo, Norway

${ }^{111}$ Division of Orthopedics, Oslo University Hospital, Oslo, Norway

${ }^{112}$ Institute of Clinical Medicine, Faculty of Medicine, University of Oslo, Oslo, Norway

${ }^{113}$ Broad Institute, Cambridge MA Harvard Medical School, Boston MA, Massachusetts General Hospital, Boston MA, USA

${ }^{114}$ National Trauma Research Institute, The Alfred Hospital, Monash University, Melbourne, Victoria, Australia

${ }^{115}$ Department of Neurosurgery, Odense University Hospital, Odense, Denmark

${ }^{116}$ International Neurotrauma Research Organisation, Vienna, Austria

${ }^{117}$ Klinik für Neurochirurgie, Klinikum Ludwigsburg, Ludwigsburg, Germany

${ }^{118}$ Division of Biostatistics and Epidemiology, Department of Preventive Medicine, University of Debrecen, Debrecen, Hungary

${ }^{119}$ Department Health and Prevention, University Greifswald, Greifswald, Germany

${ }^{120}$ Department of Anaesthesiology and Intensive Care, AUVA Trauma Hospital, Salzburg, Austria 
${ }^{121}$ Department of Neurology, Elisabeth-TweeSteden Ziekenhuis, Tilburg, the Netherlands

${ }^{122}$ Department of Neuroanesthesia and Neurointensive Care, Odense University Hospital, Odense, Denmark

${ }^{123}$ Department of Neuromedicine and Movement Science, Norwegian University of Science and Technology, NTNU, Trondheim, Norway

${ }^{124}$ Department of Physical Medicine and Rehabilitation, St.Olavs Hospital, Trondheim University Hospital, Trondheim,Norway

${ }^{125}$ Department of Neurosurgery, University of Pécs, Pécs, Hungary

${ }^{126}$ Division of Neuroscience Critical Care, John Hopkins University School of Medicine, Baltimore, USA

${ }^{127}$ Department of Neuropathology, Queen Elizabeth University Hospital and University of Glasgow, Glasgow, UK

${ }^{128}$ Dept. of Department of Biomedical Data Sciences, Leiden University Medical Center, Leiden, The Netherlands

${ }^{129}$ Department of Pathophysiology and Transplantation, Milan University, and Neuroscience ICU, Fondazione IRCCS Cà Granda Ospedale Maggiore Policlinico, Milano, Italy

${ }^{130}$ Department of Radiation Sciences, Biomedical Engineering, Umeå University, Umeå, Sweden

${ }^{131}$ Perioperative Services, Intensive Care Medicine and Pain Management, Turku University Hospital and University of Turku, Turku, Finland

${ }^{132}$ Department of Neurosurgery, Kaunas University of Health Sciences, Kaunas, Lithuania

${ }^{133}$ Intensive Care and Department of Pediatric Surgery, Erasmus Medical Center, Sophia Children's Hospital, Rotterdam, The Netherlands

${ }^{134}$ Department of Neurosurgery, Kings college London, London, UK

${ }^{135}$ Neurologie, Neurochirurgie und Psychiatrie, Charité - Universitätsmedizin Berlin, Berlin, Germany

${ }^{136}$ Department of Intensive Care Adults, Erasmus MC- University Medical Center Rotterdam, Rotterdam, the Netherlands

${ }^{137}$ icoMetrix NV, Leuven, Belgium

${ }^{138}$ Movement Science Group, Faculty of Health and Life Sciences, Oxford Brookes University, Oxford, UK

${ }^{139}$ Psychology Department, Antwerp University Hospital, Edegem, Belgium

${ }^{140}$ Director of Neurocritical Care, University of California, Los Angeles, USA

${ }^{141}$ Department of Neurosurgery, St.Olavs Hospital, Trondheim University Hospital, Trondheim, Norway

${ }^{142}$ Department of Emergency Medicine, University of Florida, Gainesville, Florida, USA

${ }^{143}$ Department of Neurosurgery, Charité - Universitätsmedizin Berlin, corporate member of Freie Universität Berlin, Humboldt-Universität zu Berlin, and Berlin Institute of Health, Berlin, Germany

${ }^{144}$ VTT Technical Research Centre, Tampere, Finland

${ }^{145}$ Section of Neurosurgery, Department of Surgery, Rady Faculty of Health Sciences, University of Manitoba, Winnipeg, MB, Canada

\section{Author contributions}

HFL and IEC planned the study, IEC, JAH, and NVL wrote the article, and IEC analyzed the data. JAH and AIRM provided insight into clinic processes. AIRM and HFL provided valuable input in the writing process and expert knowledge. All authors revised and approved the final version of the manuscript.

\section{Source of support}

The authors' work on this study is partly funded by the European Union seventh Framework Program (grant 602150) for Collaborative European Neurotrauma Effectiveness Research in Traumatic Brain Injury (CENTER-TBI). Additional support for CENTER-TBI was obtained from the Hannelore Kohl Stiftung (Germany), OneMind (USA), Integra LifeSciences Corporation (USA), and NeuroTrauma Sciences (USA).

\section{Availability of data and material}

The datasets generated and/or analysed during the current study are not publicly available because participants gave no consent for data sharing.The datasets generated and/or analysed during the current study are not publicly available because participants gave no consent for data sharing.

\section{Conflicts of interest}

All authors are (partly) funded by the CENTER-TBI study, which is in essence a comparative effectiveness research study. Findings of this study concern the use of CENTER-TBI in comparative effectiveness research, which could be noted as competing interests.

We confirm that the manuscript complies with all instructions to authors and that authorship requirements have been met and the final manuscript was approved by all authors. Further we confirm that this manuscript has not been published elsewhere and is not under consideration by another journal

\section{Ethical approval/informed consent}

The CENTER-TBI study (EC grant 602150) has been conducted in accordance with all relevant laws of the EU if directly applicable or of direct effect and all relevant laws of the country where the recruiting sites were located, including but not limited to, the relevant privacy and data protection laws and regulations (the "Privacy Law"), the relevant laws and regulations on the use of human materials, and all relevant guidance relating to clinical studies from time to time in force including, but not limited to, the ICH Harmonised Tripartite Guideline for Good Clinical Practice (CPMP/ICH/135/95) ("ICH GCP") and the World Medical Association Declaration of Helsinki entitled "Ethical Principles for Medical Research Involving Human Subjects." Informed Consent by the patients and/or the legal representative/next of kin was obtained, accordingly to the local legislations, for all patients recruited in the Core Dataset of CENTER-TBI and documented in the e-CRF. Ethical approval was obtained for each recruiting sites. The list of sites, Ethical Committees, approval numbers and approval dates can be found here: https://www.center-tbi.eu/project/ ethical-approval. The Center for Medical Decision Making, Department of Public Health, Erasmus MC as well as the Department of Neurosurgery, Antwerp University Hospital and University of Antwerp cooperated in the development and realization of the study, and therefore participating researchers had access to the data.

\section{Open Access}

This article is licensed under a Creative Commons Attribution 4.0 International License, which permits use, sharing, adaptation, distribution and reproduction in any medium or format, as long as you give appropriate credit to the original author(s) and the source, provide a link to the Creative Commons licence, and indicate if changes were made. The images or other third party material in this article are included in the article's Creative Commons licence, unless indicated otherwise in a credit line to the material. If material is not included in the article's Creative Commons licence and your intended use is not permitted by statutory regulation or exceeds the permitted use, you will need to obtain permission directly from the copyright holder. To view a copy of this licence, visit http://creativecommons.org/licenses/by/4.0/.

\section{Publisher's Note}

Springer Nature remains neutral with regard to jurisdictional claims in published maps and institutional affiliations.

Received: 26 February 2021 Accepted: 20 October 2021 Published: 6 December 2021

\section{References}

1. Stocchetti N, Carbonara M, Citerio G, Ercole A, Skrifvars MB, Smielewski P, et al. Severe traumatic brain injury: targeted management in the intensive care unit. Lancet Neurol. 2017;16:452-64.

2. Ghajar J. Traumatic brain injury. Lancet. 2000;356:923-9.

3. Maas AIR, Menon DK, Adelson PD, Andelic N, Bell MJ, Belli A, et al. Traumatic brain injury: integrated approaches to improve prevention, clinical care, and research. Lancet Neurol. 2017;16:987-1048.

4. Bragge P, Synnot A, Maas A, Menon D, Cooper DJ, Rosenfeld JV, et al. A state of the science overview of randomised controlled trials evaluating acute management of moderate to severe traumatic brain injury. J Neurotrauma. 2015;1478:1-85.

5. Lingsma HF, Roozenbeek B, Li B, Lu J, Weir J, Butcher I, et al. Large between-center differences in outcome after moderate and severe traumatic brain injury in the international mission on prognosis and clinical trial design in traumatic brain injury (IMPACT) study. Neurosurgery. 2011;68:601-7. 
6. Bulger EM, Nathens AB, Rivara FP, Moore M, Mackenzie EJ, Jurkovich GJ. Management of severe head injury: Institutional variations in care and effect on outcome. Crit Care Med. 2002;30:1870-6.

7. Maas AIR, Menon DK, Lingsma HF, Pineda JA, Sandel ME, Manley GT. Re-orientation of clinical research in traumatic brain injury: report of an international workshop on comparative effectiveness research. J Neurotrauma. 2012;29:32-46

8. Maas AIR, Menon DK, Steyerberg EW, Citerio G, Lecky F, Manley GT, et al. Collaborative European neurotrauma effectiveness research in traumatic brain injury (CENTER-TBI): a prospective longitudinal observational study. Neurosurgery. 2015;76:67-80.

9. Cnossen MC, Polinder S, Lingsma HF, Maas AIR, Menon D, Steyerberg EW, et al. Variation in structure and process of care in traumatic brain injury: provider profiles of European neurotrauma centers participating in the CENTER-TBI study. PLOS ONE. 2016;11:1-21.

10. Olsson U. Maximum likelihood estimation of the polychoric correlation coefficient. Psychometrika. 1979;44:443-60.

11. Murtagh F, Legendre P. Ward's hierarchical agglomerative clustering method: which algorithms implement Ward's criterion? J Classif. 2014:31:274-95.

12. Schwarz G. Estimating the dimension of a model. Ann Stat. 1978;6:461-4.

13. Fraley C. How many clusters? Which clustering method? Answers via model-based cluster analysis. Comput J. 1998;41:578-88.

14. Maechler M, Rousseeuw P, Struyf A, Hubert M. cluster: "Finding Groups in Data": Cluster Analysis Extended Rousseeuw et al. 2019. https://svn.rproject.org/R-packages/trunk/.

15. Wei T, Simko V. R package "corrplot": Visualization of a Correlation Matrix. 2021. https://github.com/taiyun/corrplot.
16. Nakazawa M. fmsb: Functions for Medical Statistics Book with some Demographic Data. 2021. http://minato.sip21c.org/msb/.

17. Wickham H, François R, Henry L, Müller K. dplyr: A Grammar of Data Manipulation. 2020. https://cran.r-project.org/package=dplyr.

18. Chang W. webshot: Take Screenshots of Web Pages. 2019. https://github. com/wch/webshot/.

19. Kolde R. pheatmap: Pretty Heatmaps. 2019. https://cran.r-project.org/ package $=$ pheatmap.

20. Neuwirth E. RColorBrewer: ColorBrewer Palettes. 2014. https://cran.r-proje ct.org/package $=$ RColorBrewer.

21. R Core Team. R: A Language and Environment for Statistical Computing 2016.

22. Carney N, Totten AM, O'Reilly C, Ullman JS, Hawryluk GWJ, Bell MJ, et al. Guidelines for the management of severe traumatic brain injury. Neurosurgery. 2017;80:6-15.

23. Steyerberg EW, Wiegers E, Sewalt C, Buki A, Citerio G, De Keyser V, et al. Case-mix, care pathways, and outcomes in patients with traumatic brain injury in CENTER-TBI: a European prospective, multicentre, longitudinal, cohort study. Lancet Neurol. 2019;18:923-34.

24. Huijben JA, Wiegers EJA, Lingsma HF, Citerio G, Maas AIR, Menon DK, et al. Changing care pathways and between-center practice variations in intensive care for traumatic brain injury across Europe: a CENTER-TBI analysis. Intensive Care Med. 2020;46:995-1004.

25. Van Eure A, de Ragu CW, Caur HKP. Neurosurgical treatment variation of traumatic brain injury: evaluation of acute subdural hematoma management in belgium and the Netherlands. J Neurotrauma. 2017;34:881-9. 